\title{
Review: some interventions are effective in reducing medical errors
}

\author{
Ioannidis JP, Lau J. Evidence on interventions to reduce medical errors. An overview and recommendations for future \\ research.J Gen Intern Med 2001 May;16:325-34.

\section{QUESTION: What is the effectiveness of interventions specifically aimed at reducing medical errors?}

\section{Data sources}

Studies in any language were identified by searching Medline (1966 to 2000) and EMBASE with the terms medical errors, prescription errors, diagnostic errors, and medication errors, and terms associated with randomised controlled trials. Additional studies were identified by screening the Cochrane Clinical Trials Registry and bibliographies of relevant studies and by communicating with experts and colleagues.

\section{Study selection}

Studies were selected if they were randomised controlled trials, compared an intervention with placebo or no intervention, and specified the aim of reducing medical errors as a primary or secondary outcome. Studies were excluded if they emphasised patient compliance, they evaluated omission of orders or actions suggested by preventive medicine, the only outcome was interrater variability in the absence of a gold standard, the intervention was a different imaging or laboratory test to improve diagnosis beyond the diagnostic technology in the control group, trainees and patients were involved rather than professional staff, fictitious or simulated cases was considered, or response time but not errors were evaluated. Some non-randomised studies were also evaluated for comparison of pertinent data.

\section{Data extraction}

Data were extracted on sample size, study setting, study population characteristics, quality components, definition of errors, whether reducing error was a primary or secondary outcome, number of errors per arm, and comparative statistics.

\section{Main results}

52 studies were retrieved, and 13 randomised studies met the inclusion criteria. 3 studies involved $>1000$ patients, and $2 \mathrm{had}>1000$ opportunities for error. The study settings and data collection methods varied among all the studies, and only 1 study met all 4 criteria for high quality research. The studies assessed errors related to medication, prescription, dosage, diagnosis, management, and recognition of the seriousness of illness. In 9 studies, the interventions reduced errors (range of errors $0 \%$ to $37 \%$ ) compared with a control group ( $4 \%$ to $63 \%)$. In the other 4 studies, the intervention did not reduce the error rate, but the study authors reported favourable aspects of the intervention. The error rate was increased in 1 study because of the intervention-a protocol for assessing the need for radiography after lower extremity injury - but it also reduced emergency waiting time. 21 of 24 nonrandomised studies found a reduction in errors with the intervention.

\section{Conclusions}

Some interventions aimed at reducing medical errors are effective in decreasing medical error rates in various settings. However, few high quality studies of such interventions exist.

\section{COMMENTARY}

The review by Ioannidis and Lau systematically identifies and assesses the published research on interventions to reduce medical errors. Its publication is timely given the unprecedented concern about patient safety in the wake of the Institute of Medicine report on medical error ${ }^{1}$ and the pressure on healthcare providers to reduce errors by using computerised physician order entry and other interventions. $^{2}$

The review provides a good news-bad news message for providers trying to reduce errors. The good news is that a few well-studied interventions decrease medical errors. The bad news is that only 13 randomised controlled trials assessed the effectiveness of these interventions, and none of these trials measured reductions in morbidity or mortality.

Some highly touted interventions for reducing errors are notably absent. Computerised physician order entry, simulation, team training programmes, root cause analysis, bar coding, and error reporting systems have not been shown to reduce errors in randomised controlled trials. These findings are of concern given the pressure on providers to adapt these methods, even though they may be costly and time consuming and may expose providers to liability.

A recent report ${ }^{3}$ took a different perspective by looking for evidence-based practices that improve patient safety rather than the related, but more focused, search for interventions to prevent specific errors. The study identified 11 well-studied practices that can improve patient safety, such as antibiotic prophylaxis in surgical patients to prevent postoperative infections.

The complexity of medical errors and the proposed interventions mean that research in the field is difficult and may proceed slowly. In the meantime, providers should be wary of using inadequately tested interventions to reduce medical errors.

Eric Thomas, MD, MPH University of Texas-Houston Medical School Houston, Texas, USA

1 Kohn LT, Corrigan JM, Donaldson MS, eds. To err is human: building a safer health system. Committee on quality health care in America, Institute of Medicine. Washington, D.C.: National Academy Press, 2000.

2 The Leapfrog Group for patient safety. (http:// www.leapfroggroup.org/index.html)

3 Making health care safer: a critical analysis of patient safety practices. Evidence report/technology assessment no. 43. AHRQ publication no. 01-E058. Rockville, MD: Agency for Healthcare Research and Quality, July 2001. http:// www.ahrq.gov/clinic/ptsafety/index.html.

1384 